7. Burdy G, Panis Y, Alves A, Nemeth J, Lavergne-Slove A, Valleur P. Identifying patients with T3-T4 node negative colon cancer at high risk of recurrence. Dis Colon Rectum. 2001;44:1682-8.

8. Morales-Gutierrez C, Vegh I, Colina F, Gomez-Camara A, IgnacioLanda J, Ballesteros D, et al. Survival of patients with colorectal carcinoma: possible prognostic value of tissular carbohydrate antigen 19.9 determination. Cancer. 1999;86:1675-81.

9. Moriya Y. Treatment strategy for locally recurrent rectal cancer. Jpn J Clin Oncol. 2006;36:127-31.

10. Tanaka K, Noura S, Ohue M, Seki Y, Yamada T, Miyashiro I, et al. Doubling time of carcinoembryonic antigen is a significant prognostic factor after the surgical resection of locally recurrent rectal cancer. Dig Surg. 2008;25:319-24.

\section{Blue rubber bleb nevus syndrome - Role of aggressive surgical resection}

\section{Introduction}

Blue rubber bleb nevus syndrome is a rare multifocal venous malformation primarily involving the skin, soft tissues and the gastrointestinal tract but may involve any tissue. We present the case of a young girl who developed recurrent melena necessitating surgical resection.

\section{Case report}

The patient was a 12-year old girl, a known case of blue

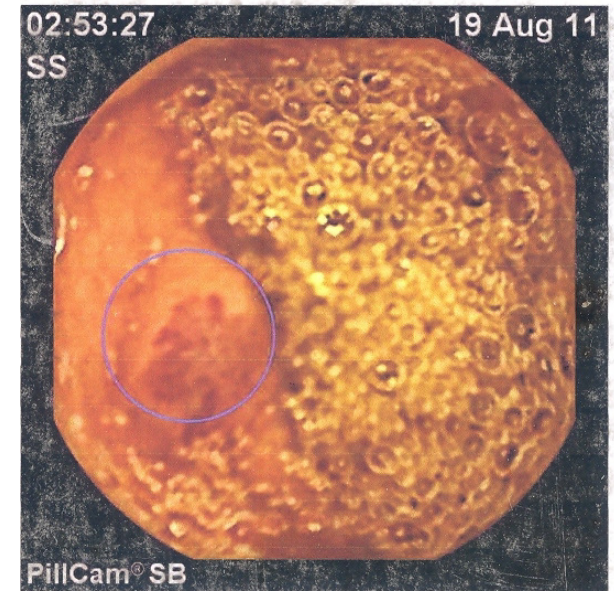

Figure 2 Capsule endoscopy revealing intraluminal blebs rubberbleb nevus syndrome (BRBNS) who had numerous lesions (Figure 1) numbering 65, over scalp, lips, trunk, abdominal wall, all four extremities, gluteal region and vulva for which she had received four sittings of injection sclerotherapy (sodium tetradecyl sulphate) with satisfactory regression of the lesions injected.

She developed intermittent episodes of small volume melena over the one year prior to presentation, leading to anemia and chronic fatigue necessitating ten blood transfusions. Blood pool scan showed no evidence of active intestinal bleed but showed multiple pooling in the intestine along with numerous musculoskeletal uptakes.

Wireless capsule endoscopy (Figure 2) revealed multiple intraluminal blebs from duodenojejunal flexure to the cecum with no active bleed.

In view of recurrent episodes of melena requiring multiple blood transfusions, surgery was planned. On examination she had pallor. Abdominal examination was unremarkable. The abdomen was accessed through a right upper transverse

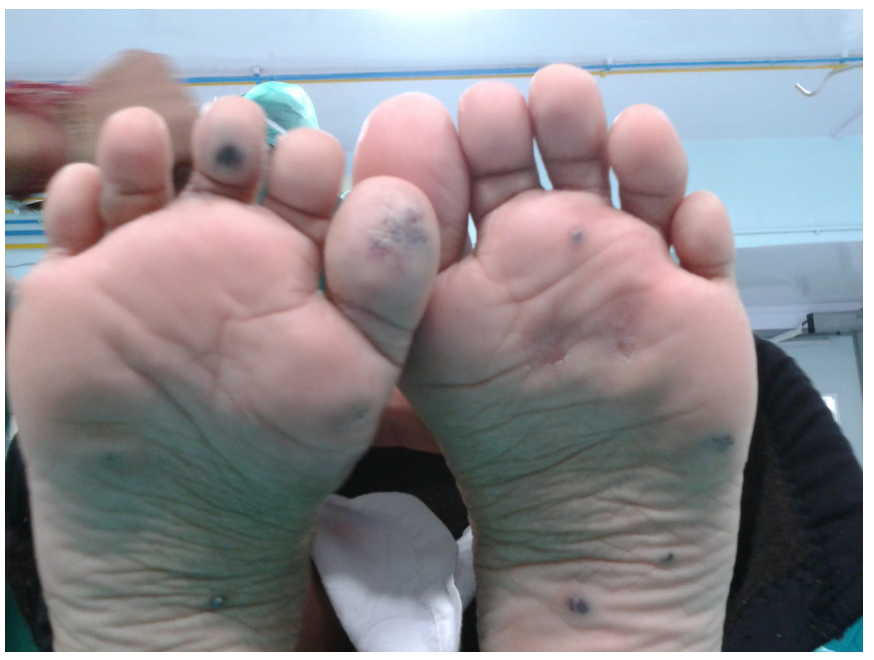

Figure 1 Cutaneous manifestation of BRBNS

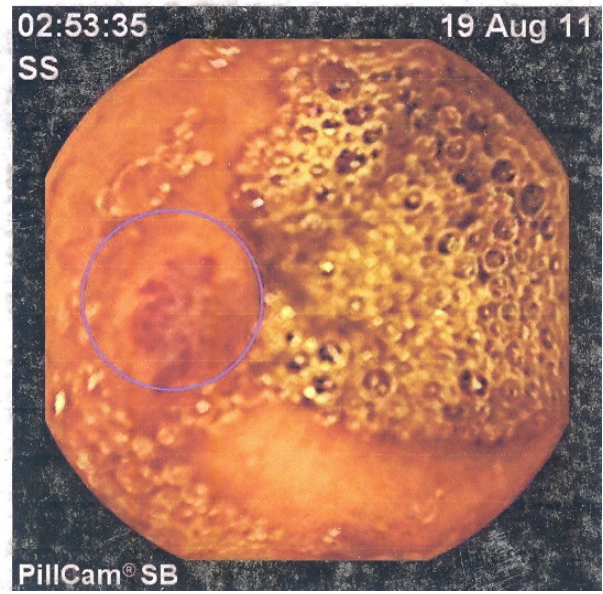




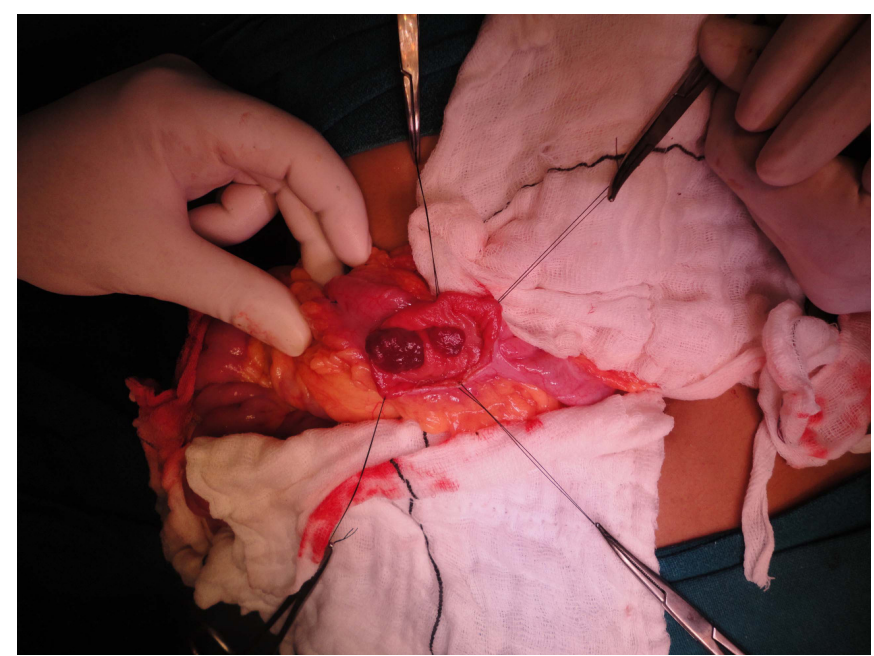

Figure 3 Intraoperative picture of intraluminal blebs

incision. Peroperatively, a total of 54 blebs were seen scattered all over the small intestine from duodenojejunal flexure to the terminal ileum and 12 blebs were observed between the cecum and descending colon. All the blebs were a mix of transmural, mucosal and submucosal (Figure 3) lesions.

Complete excision of all the intestinal blebs using a combination of wedge resections, bowel resections, multiple enterotomies with intussusceptions of proximal and distal bowel, followed by suture ligation and polypectomy was done in a single sitting lasting over 7 hours. In addition 3 wedge resections of liver lesions along with gastrotomy and removal of a large lesser curvature lesion were also carried out.

The postoperative period was uneventful. The child was allowed oral intake on day 5 and was discharged on day 10 . She has been completely asymptomatic 24 months following surgery. The histopathology report showed cavernous hemangiomas formed by dilated capillaries with flat endothelial cells and connective tissue stroma compatible with BRBN lesion.

\section{Discussion}

William Bean characterized the blue rubber bleb nevus syndrome in 1958, ${ }^{1}$ earning the eponym "Bean syndrome". Typically, venous malformations of the skin are small $(1-3 \mathrm{~cm})$ blue "nipples" or "buttons" that are covered by skin and feel hard or rubbery, hence the name "blue rubber bleb nevi ".

Though the skin lesions range from several to hundreds, it is the gastrointestinal tract lesions that are of clinical relevance due to their propensity to hemorrhage.The bleeding is characteristically from the small bowel and is slow and continuous rather than massive and life threatening. ${ }^{1}$ Wireless capsule endoscopy is effective in identifying obscure causes of bleed from the small intestine beyond the ligament of Treitz. ${ }^{2}$ However, being non therapeutic in nature, its use is limited in pediatric gastroenterology.

Cutaneous lesions are best managed with sclerotherapy using ethanol or sodium tetradecyl sulphate. Our patient responded remarkably well with multiple sittings of sclerotherapy. Though there are isolated reports of gastrointestinal lesions managed by endoscopic sclerotherapy ${ }^{3}$ and polypectomy, ${ }^{4}$ the risk of perforation, necrosis and incomplete treatment always exists. Moreover, extensive lesions and transmural lesions cannot be tackled endoscopically.

Aggressive surgical resection, as in our patient, comprising wedge resections, small bowel resections, suture ligation and polypectomy via enterotomies is the main stay of cure for extensive lesions and transmural intestinal lesions that continue to bleed. Given the assumption that completely resected lesions will not recur, surgery provides a permanent cure and rids the patient of long term iron supplements and follow up. Fishmanetal ${ }^{5}$ showed their experience with complete and extensive surgical resection in 10 patients with excellent results. Though only 9 reports exist in literature involving extensive surgical resection for these gastrointestinal tract vascular lesions; this is only the second report documenting such an extensive surgical resection in a child.

In conclusion, blue rubber bleb nevus syndrome is a rare clinical entity with numerous cutaneous and gastrointestinal tract vascular lesions. The cutaneous lesions can be adequately managed with multiple patient sittings of sclerotherapy. The intestinal lesions require a more aggressive surgical approach. Though the surgery may be demanding and tedious, a good multidisciplinary approach with adherence to standard surgical principles results in successful outcome with minimal morbidity.

V SHANKAR RAMAN, VEERESHWAR BHATNAGAR

Correspondence: Dr. V Bhatnagar Department of Pediatric Surgery All India Institute of Medical Sciences New Delhi- 110029, India

Email: veereshwarb@hotmail.com

\section{References}

1. Bean WB. Blue rubber-bleb nevi of the skin and gastrointestinal tract. In: Bean WB. Vascular Spiders and Related Lesions of the Skin. Springfield, IL: Charles C Thomas; 1958:17-185.

2. Hartmann D, Schmidt H, Bolz G, Schilling D, Kinzel F, Eickhoff 
A, et al. A prospective two-center study comparing wireless capsule endoscopy with intraoperative enteroscopy in patients with obscure GI bleeding. Gastrointest Endosc. 2005;61:826-32.

3. Sala Felis T, Urquijo Ponce JJ, LópezViedma B, Pertejo Pastor $\mathrm{V}$, BerenguerLapuerta J. Blue nevus syndrome: endoscopic treatment by sclerosis and band ligation. Gastroenterol Hepatol. 1999;22:136-8.

4. Mohammad Hassan Emami, SaeidHaghdani, Hamid Tavakkoli, ParvinMahzounil.Endoscopic polypectomy resection of blue rubber bleb nevus lesions in small bowel. Indian J Gastroenterol. 2008;27:165-6.

5. Steven J. Fishman, C. Jason Smithers, Judah Folkman, Dennis P. Lund.Blue Rubber Bleb Nevus Syndrome- Surgical Eradication of Gastrointestinal Bleeding. Ann Surg. 2005;241:523-8.

\section{EHPVO presenting as Chronic myeloid leukemia immediately post splenectomy}

\section{Introduction}

Non-cirrhotic portal fibrosis (NCPF) and extra-hepatic portal venous obstruction (EHPVO) are important causes of noncirrhotic PHT. The most common cause in EHPVO patients leading to PHT is portal vein thrombosis (PVT). Various mechanisms for PVT have been identified like portal vein injury, congenital defects, hypercoagulable states etc. ${ }^{1}$ Hypercoagulable states may be due to inherited gene mutations $^{2}$ or acquired causes. Acquired causes include use of oral contraceptives, pregnancy and myeloproliferative disorders(MPDs). Both latent and occult MPDs are known to constitute an important cause of PVT. ${ }^{3}$

We report the case of a 30-year old male patient with EHPVO who underwent successful shunt surgery and splenectomy with sudden precipitation of myeloproliferative disorder (MPD), post splenectomy.

\section{Case presentation}

A30-year old male patient presented to the outpatient department of our institute in October 2013 with complaints of generalized weakness, pain in right upper quadrant, jaundice with high colored urine, pruritis, and intermittent clay-colored stools; all symptoms spanning a four-month period prior to presentation.On examination the patient had icterus with hepatosplenomegaly. Vitals were within normal limits.Patient had a significant past history.He was a known case of EHPVO diagnosed in 2008, non-bleeder (status EVL [endoscopic variceal ligation] done in 2010) with PHT.Upper GI endoscopyshowed grade 2 esophageal varices. CECT revealed chronic portal vein thrombosis with multiple porto-systemic collaterals and splenomegaly.MRI (abdomen) done in July2013 reported EHPVO with portal cavernoma , CBD compression and IHBRD, consistent with portal biliopathy.Liverbiopsy also carried out in November 2013 showed chronic obstructive biliary pathology with portal fibrosis (consistent with portal biliopathy) and an occasional focus of extramedullaryhematopoiesis (EMH) (Figure 1).Complete blood counts $[\mathrm{CBC}]$ were within normal limits.Liver Function tests[LFTs] were derangedand kidneyfunction testswere within normal limits.On the basis of clinical history, past history, examination and investigations the patient was diagnosed as:"EHPVO with PHT, asymptomatic hypersplenism, symptomatic portal biliopathy and surgical obstructive jaundice."In view of the above diagnosis, the patient was planned for splenectomy with proximal splenorenal shunt. In Oct 2013, the patient underwent successful shunt surgery Specimen pathology revealedfibrocongestive spleen with occasional foci of EMH (Figure 2). LFT showed improvement

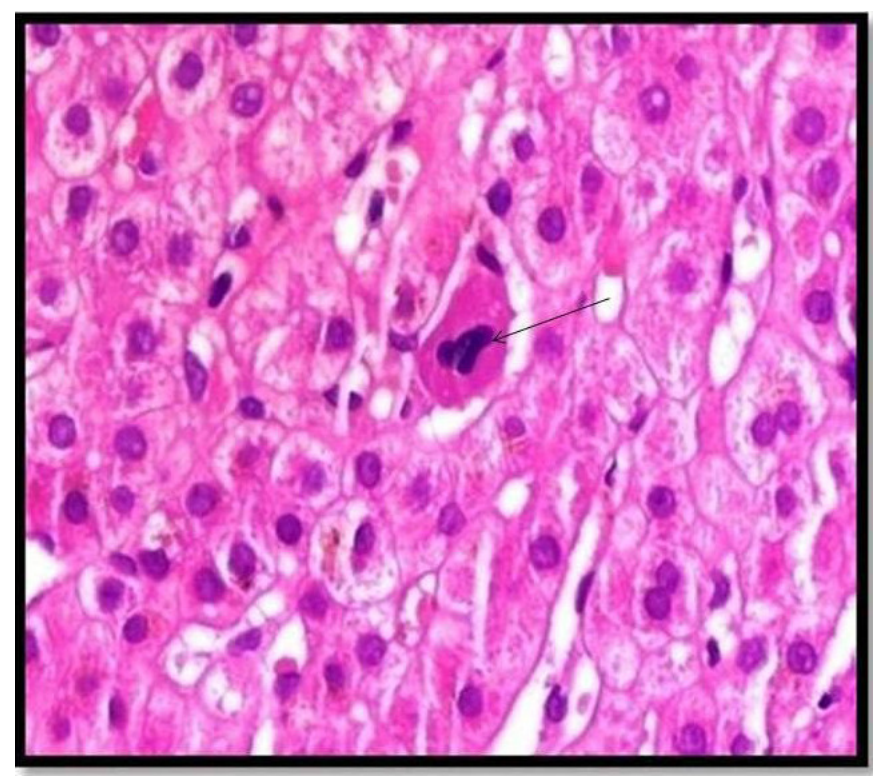

Figure 1: Liver biopsy showing an occasional megakaryocyte [black arrow] (HE Stain, oil emersion) 